\title{
La non-commercialisation du génome humain
}

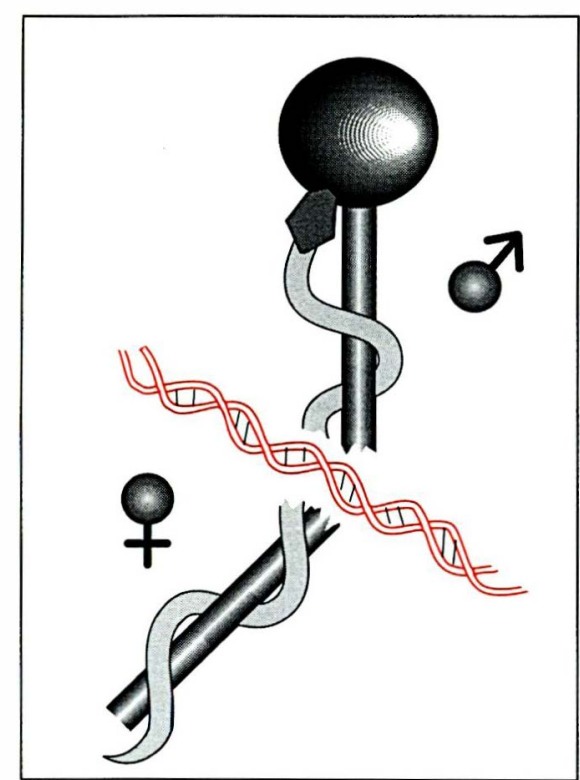

Comité consultatif national d'ćthique pour les sciences de la vie et de la santé, 101 ruc de Tolbiac, 75654 Paris Cedex 13, France.

\section{Préambule}

Problèmes éthiques de la commercialisation du génome humain

Le projet Génome Humain doit avoir pour finalité les progrès dans la connaissance, la santé et la qualité de la vie.

Dès le début, les différents protagonistes du programme ont insisté sur l'absolue nécessité d'une circulation très rapide et libre de l'information. Mais le 20 juin 1991, Craig Venter, travaillant au NIH (National Institutes of Health), a déposé au bureau américain des brevets (US Patent Office) une demande de brevets concernant 337 " nouveaux gènes humains ". Dans un premier temps, ce laboratoire se contente de déterminer la séquence d'environ 250 lettres de l'ADN complémentaire ce qui est largement suffisant pour fabriquer les outils permettant d'isoler les gènes correspondans. A ce stade on ne sait pas ce qu'est le gène, ce pourquoi il code et ce qu'il fait.

La puissance des équipements disponibles pourrait ainsi conduire à des demandes de brevets concernant des milliers de gènes humains potentiels : les "gènes nus" (naked genes).

Il convient d'analyser les processus de protection des inventions et de la diffusion du savoir et leurs applications au génome.

\section{- Les brevets}

Il faut d'abord noter que·la législation sur le brevet a été (et pourrait être) évolutive en fonction des progrès et qu'il existe de profondes différences entre l'Europe et les États-Unis. Trois critères de brevetabilité sont retenus.

\section{La nouveauté}

Une différence importante existe entre l'Europe et les États-Unis.

- En Europe, la date de dépôt de la demande doit être antérieure à toute publication.
- Aux États-Unis, il existe un délai d'un an entre la date "d'invention" (publication) et la date de dépôt.

\section{L'inventivité}

Il faut une activité inventive qui fait l'originalité du travail.

Dans ce sens, on peut se demander si répond à ce critère le dépôt de demande de brevets du NIH sur des séquences humaines d'ADN complémentaires, car ce travail peut être fait à partir de banques d'ADN disponibles et en utilisant une technologie à la portée de tout laboratoire correctement équipé dans ce but.

\section{L'application industrielle}

La demande de brevet doit préciser les applications potentielles de l'invention.

Sur ce point, des différences existent entre les États-Unis et l'Europe : les États-Unis prennent en compte la notion d'utilité de l'invention, alors que les offices européens considèrent son application industrielle.

Par ailleurs, il est possible, aux ÉtatsUnis, par le biais de continuation-inpart (CIP), d'apporter de la matière nouvelle à l'invention pour la conforter et en étendre le domaine d'application.

Dans le cas des demandes du NIH pour breveter des séquences d'ADN complémentaires, on évoque leur fonction probable, car elles ont sélectionné des séquences d'ADN qui s'exprimeraient (sans que cela soit démontré) dans des tissus cérébraux et donc pourraient avoir une application dans des affections neurologiques (sans plus de précision).

Il convient de bien différencier une découverte d'une invention. L'article 52 2a de la CBE (Convention sur le Brevet Européen) énonce :

- " ne sont pas considérées comme des inventions, les découvertes";

- la découverte se définit : " comme l'acte, le procédé, ou la circonstance par lequel on acquiert la connaissance de quelque chose d'inconnu ou de 
non reconnu jusqu'alors ", donc préexistant.

Nouveauté, inventivitć et application concrète représentent donc les critères de la distinction entre la déconverte et l'invention.

On peut aussi rappeler l'article 53a de la $\mathrm{CBE}$ qui précise que les inventions dont la publication ou la mise en œuvre serait contraire à l'ordre public ou aux bonnes mœurs sont exclues de la brevetabilité.

Le brevet est aussi un moyen de diffusion du savoir :

- au moment du dépôt, on doit mettre à la disposition de la communauté scientifique les éléments permettant de démontrer la reproductibilité de "l'invention": souche de microorganismes, de cellules, ou formule détaillée d'une séquence d'ADN ;

- à l'expiration des 18 mois, tous les documents sont disponibles dans une banque de données sur les brevets. C'est donc une source importante d'informations.

\section{- Les banques de données}

La masse des informations engendrée par les recherches du projet Génome Humain et leur diffusion ne peuvent plus se faire par les publications scientifiques. Seul un support informatique pourra gérer cette information dans des banques de données.

Toute recherche dans le cadre du génome humain doit avoir accès à des bases de données. La mise au point et la possession d'un tel outil donne un avantage réel pour les chercheurs et peut conduire à la protection de données au profit d'une industrie biotechnologique.

La question de l'accès aux données se pose donc au plan individuel et au plan collectif. Ceux qui ont investi massivement temps et argent dans la confection de ces outils accepteront-ils un libre accès de compétiteurs chercheurs ou industriels. Il serait non éthique qu'un retard non justifié dans la diffusion des connaissances entraîne des retards dans les applications thérapeutiques possibles.

La question de l'accès aux bases de données est liée directement à celle de la " propriété du savoir ". C'est donc un problème fondamental de l'éthique de la recherche.

Cette question peut être interprétée à $\mathrm{m} / \mathrm{s} n^{\circ} 10$, vol. 7, décembre 91 la lumière de l'article 27 de la Déclaration Universelle des Droits de l'Homme. " Toute personne a le droit de prendre part librement à la vie culturelle de la communauté, de jouir des arts et de participer aux progrès scientifiques et aux bienfaits qui en résultent " (section 1) complétée par la section 2 de ce même article " chacun a droit à la protection des intérêts moraux et matériels découlant de toute production scientifique, littéraire ou artistique dont il est l'auteur. "

\section{Considérations éthiques}

Dans le cadre du génome humain, comment concilier les principes de non commercialisation du corps humain, les données de la biologie et les aspects juridiques et administratifs de brevetabilité et de gestion des banques de données?

- Des limites biologiques ne peuvent pas être fixées sur des critères nets

Dans le corps humain et ses éléments, on passe progressivement du corps en entier, aux organes, aux tissus (le sang par exemple), aux cellules (les spermatozoïdes par exemple), aux gènes, aux messagers cellulaires (ARN messager), aux protéines.

Les protéines peuvient être l'objet de commercialisation et de brevets pour les procédés de leur obtention (insuline, hormone de croissance, érythropoiétine, interleukines...) et pour leurs applications.

Les messagers sont labiles, mais à partir des messagers on peut définir des séquences d'ADN complémentaire, copies de l'information de l'ADN d'un gène. Ces $\mathrm{ADN}$ copies n'existent pas naturellement, ce sont des substances chimiques artificielles. Ils peuvent être utilisés pour des tests diagnostiques, ou comme premier temps de la production de protéines à action thérapeutique par exemple.

- Fixer des limites éthiques serait plus opportun en répondant aux questions : quelles sont les menaces au plan de l'éthique?

C'est cette démarche qui a été retenue déjà par le Comité Consultatif National d'Éthique (CCNE) pour l'embryon où la définition n'a pas été biologique mais éthique : "personne humaine potentielle ", dans la crainte, en particulier, de fabriquer des personnes humaines à la carte.
Aujourd'hui apparaît la menace d'une appropriation dans un but lucratif d'informations sur le génome humain, patrimoine de l'humanité, et à une appropriation du savoir qui pourrait devenir un monopole réservé au développement de biotechnologies.

Ces appropriations peuvent être comparées à la mise de son drapeau sur une terre non encore explorée.

De nombreux scientifiques impliqués dans le séquençage des gènes humains considèrent que leur activité, soutenue par des fonds publics ou par des organisations caritatives, est un Service pour la communauté, que les séquences d'ADN complémentaires ainsi que d'autres éléments du génome (les microsatellites par exemple) constituent une information qui doit être librement disponible, et ne peuvent être l'objet d'une appropriation, à travers un brevet ou une limitation de l'accès à unc banque de données.

Devant ces nouvelles menaces éthiques, un nouvel avis du CCNE devient nécessaire.

\section{Avis sur Ia non-commercialisation du génome humain}

Le Comité Consultatif National d'Éthique s'est régulièrement soucié des problèmes éthiques relatifs aux progrès des connaissances en génétique et aux applications de ces progrès :

En 1985, un avis sur les problèmes posés par la diagnostic prénatal et périnatal.

En 1989, un avis sur la diffusion des techniques d'identification par analyse de l'ADN (technique des empreintes génétiques).

En 1990, un avis sur la thérapie génique.

En 1991, un avis sur l'application des tests génétiques aux études individuelles, études familiales, et études de population (problèmes des "banques" de l'ADN, des "banques" de cellules et de l'informatisation des données).

En outre, un groupe de travail prépare une réflexion sur l'eugénisme. Une séance des prochaines journées annuelles (18 décembre 1991) sera consacrée à cette réflexion.

Enfin, à la demande du ministre de 
la Recherche, un document sur les considérations éthiques du programme génome humain va être rendu public prochainement.

C'est un événement récent, le dépôt d'une demande de brevets sur une série de gènes humains, qui conduit le CCNE a émettre le présent avis qui précise l'application au génome humain de l'avis de 1990 sur la noncommercialisation du corps humain. De plus, cet avis sera un des éléments du rapport final sur le thème "éthique et argent".

Derrière les objectifs altruistes du programme Génome Humain et son évidente importance en termes d'acquisition des connaissances, d'applications dans le domaine de la santé, apparaissent d'autres objectifs, liés à la compétition industrielle, aux conséquences redoutables sur le plan de l'éthique : les gènes détectés sont non seulement des informations utiles pour le monde scientifique, mais aussi des données de base pour des réalisations industrielles futures à travers des prises de brevets de séquences d'ADN ou des monopoles d'utilisation des informations contenues dans des banques de données. Le brevet protégeant des fractions d'ADN apparaît dans les conditions où il est revendiqué, comme constituant un détournement des conditions normales et éthiquement admissibles, en vue d'obtenir un avantage économique injustifié.

La différence entre découverte et invention trouve là une exemplaire application.

Sur le plan de l'éthique, le problème posé par l'application des règles sur le brevet au génome humain se situe au confluent de principes auxquels le Comité attache une importance fondamentale.

L'un, sur lequel le CCNE a nettement pris position, est le principe intangible de la non-commercialisation du corps humain.

L'autre conduit à constater, à propos de cette affaire, que l'ensemble de l'information contenue dans le génome humain appartient au patrimoine commun de l'humanité : c'est un domaine de la connaissance qui ne peut faire l'objet de monopole.

Il est possible d'aller dans le sens de cette double exigence en s'appuyant sur la position suivante : les séquences d'ADN, codantes ou noncodantes, ne sont pas brevetables, elles doivent être considérées comme une information et déposées dans des banques de données accessibles à toute la communauté scientifique.

Des organisations internationales pourraient apporter leur concours à cette protection de la connaissance contre les dangers de monopolisation. Ces principes n'excluent pas, pour autant, la protection brevetaire des produits ou procédés issus de ces bases de données lorsqu'elles sont le résultat d'une réelle inventivité et d'applications convenablement décrites et dont le caractère original a été prouvé 